\title{
The Relevance of Exercise with DJ-1 Protein to Reduce Motor Symptoms and Reverse the Early Onset of Idiopathic Parkinson's Disease
}

\author{
Debjyoti Talukdar ${ }^{1}$, Sanju Aggarwal ${ }^{1}$ and Sunil Aggarwal2* \\ ${ }^{1}$ All Saints University College of Medicine (ASUCOM), St. Vincent, New York, USA \\ ${ }^{2}$ All Saints University College of Medicine (ASUCOM), New York, USA
}

*Corresponding author: Sunil Aggarwal, All Saints University College of Medicine, New York, Tel: 7176221264; Email: drsunilaggarwal@gmail.com

\section{Mini Review \\ Volume 3 Issue 4}

Received Date: November 09, 2018

Published Date: November 23, 2018

DOI: $10.23880 /$ apct- 16000142

\begin{abstract}
Parkinson's Disease (PD) is a condition which involves impairment due to deterioration of nerve cells or neurons which produce an essential chemical called dopamine. It is associated with body movement along with nerve functioning \& muscle coordination. Dopaminergic neurons are susceptible to degeneration in Parkinson's Disease as massive cell death occurs in the substantia nigra. The relevance of DJ-1 protein is quite important to the pathogenesis of Parkinson's Disease. Inactivation of DJ-1 protein is related to oxidative stress. Biochemical studies suggest that environmental toxins and pesticides can cause oxidative defects in mitochondrial complex I resulting to neuropathological and neurochemical features of Parkinson's Disease eventually leading to inactivation or absence of DJ-1 protein. Exercise alters the neuroprotective function of the DJ-1 protein by improving motor functions in Parkinson patients. Decreased DJ-1 protein concentration levels can eventually lead to motor dysfunctions resulting in tremors, rigidity \& bradykinesia. Regular exercises involving medical Qigong for kidney, liver, neck along with meditation can play a leading role in reducing the motor symptoms of Parkinson's Disease. This review will help us to understand the critical relationship between the relevance of exercise and neuroprotective effects of DJ-1 protein in idiopathic Parkinson's Disease.
\end{abstract}

Keywords: Parkinson's Disease; DJ-1 protein; Neurochemical

Abbreviations: TCM: Traditional Chinese Medicine; PD: Parkinson's Disease; TH: Tyrosine Hydrolase.

\section{Introduction}

Parkinson is often regarded as a degenerative disease with neuronal loss and reduction of dopamine production. Structured treatment is required which will enhance the neuroprotective effects and enhance the motor functions of Parkinson's Disease [1,2]. Significant reduction of the DJ-1 protein can lead to neurodegenerative disorder and eventually lead to progression of PD [3]. Qigong exercises along with acupressure can improve bodily functions of Parkinson patients such as balance, muscle hardness, and hand-eye coordination. It is recommended that PD patients should exercise regularly as it is crucial towards 


\section{Advances in Pharmacology and Clinical Trials}

improving the level of DJ-1 protein in muscles. Unconventional therapies like Qigong exercises have proven more significant in terms of peripheral and central motor aspect improvement [4-12].

Parkinson's Disease is termed as a degenerative and progressive disease as studies suggest that dopaminergic neurons are vulnerable to environmental toxicants [13]. This tends to worsen the disease as time passes. But, exercises like walking, dancing, brain vibration chanting has shown noteworthy improvement in terms of postural stability and balance. Motor functions in a retrogressive disease like Parkinson's have decreased in repetitive actions like tremor, slurring and muscle stiffness [14]. It is seen that patients suffering from PD have a history of insufficient rest, overwork, anxiety, and stress. Various blinded studies in clinical trials have proved to eliminate bias in the studies [15-17]. Holistic healing with meditation has proven helpful in alleviating people from PD. Notable exercises mentioned within the framework of Traditional Chinese Medicine (TCM) like Kidney and Liver Wind deficiency has indeed lowered the frustration and resentment levels among PD patients. Dietary consideration like lowering the consumption of greasy and sweet foods balances the body's natural rhythm. Most importantly, regular exercising has shown motor and cognitive improvement which may slow the progression of the disease.

It was reported that idiopathic Parkinson's Disease has relevance to DJ-1 protein and alpha-synuclein. DJ-1 protein prevents abnormal deposition of alpha-synuclein in neurons. Levodopa is typically administered to treat symptoms of Parkinson's Disease. It is converted to dopamine in the brain cells which renders temporary relief for Parkinson's patients. But, PD patients practicing neck exercises, acupressure, meditation with vegetarian diet have shown considerable beneficial effects in terms of reducing PD symptoms like rigidity, bradykinesia, resting tremors, pill-rolling movements, stooped posture, impaired balance \& coordination etc. [18]. However, it is noted that drugs involving levodopa do not bring a permanent cure to the patient instead the condition deteriorates after prolong use [19]. Also, it was found that balance is a critical aspect of Parkinson's Disease as patients with increased head displacement suffered from worsening balancing disorder with increased dosage of levodopa [20]. Levodopa worsens the tremor and can cause progressive rigidity and disability. It only offers temporary symptomatic relief which lasts for a couple of hours, also known as "dopa effect" [21]. On the other hand, PD patients practicing Yin Tui Na with brain vibration chanting exercises have shown prolonged effects in terms of reduction of muscle rigidity, impaired postural instability with improvement in speech and handwriting.

\section{The Relevance of Exercise, A-Synuclein and DJ-1 Proteins in Parkinson's Disease}

The unusual mutation of $\alpha$-synuclein (Alpha-Synuclein) [SNCA] gene can trigger early onset of Parkinson Disease. It was also reported that people suffering from Parkinson's Disease possess dominant trait with triplication of the $\alpha$-synuclein gene leading towards the progression of the disease [22]. The neuropathological cause for Parkinson's Disease involves the accumulation of $\beta$-amyloid $(A \beta)$ and $\alpha$-synuclein in the cerebral region of the brain [23]. Walking and running have shown to improve the DJ-1 protein concentration in plasma which prevents abnormal protein deposition of $\alpha$-synuclein in the brain. Studies performed employing lean six sigma on clinical trials with Parkinson's Disease and exercise improvements have added immensely to our knowledge about performing good clinical trials with the help of lean six sigma [24]. DJ-1 protein prevents the altered state of neurofilament in the substantia nigra region of the brain caused due to $\alpha$-synuclein and Lewy bodies which conglomerate in the neuron of Parkinson's patients [25].

The immensity of exercises like sitting zazen, Jin Shin Jyutsu, saying affirmations and prayers are not age specific but it can be instrumental towards improving the symptoms upon those impacted with the disease [26]. It is seen that these exercises have the potential to prevent early onset of the disease and reduce the depletion of a nigral nerve cell.

As per recent studies conducted, it is said that the clumping of fibers namely synuclein molecules clumps instead of being soluble in nerve cells. But, in a significant number of PD cases, increased concentration of DJ-1 protein can prevent clumping and distortion of mitochondrial complex wherein the $\alpha$-synuclein is localized.

It is contended that environmental toxins specifically pesticides play a significant role towards clumping of $\alpha$ synuclein protein. It also decreases the concentration of DJ-1 proteins resulting in partial loss of motor movements in the basal ganglia region of the brain. Breathing and neck exercises such as turning head from side to side have shown a significant reduction in muscle rigidity in Parkinson's patients. But, chronic exposure to pesticides can cause hyperkinesia and replicate the 


\section{Advances in Pharmacology and Clinical Trials}

neurochemical features of PD resulting in accumulation of $\alpha$-synuclein in the nigral neurons [27].

\section{Effects of Active Exercise and Slow Progression of Parkinson's Disease}

Motor functions have improved remarkably for patients suffering from Parkinson's Disease with recommended exercises involving medical Qigong exercise for kidney, liver, neck, standing \& balance exercises along with meditation, brain vibration therapy, yin tuina, jin shin Jitsu and neural electrical impulse with acupressure [28]. As per recent studies, it is noticed that DJ-1 protein concentration increases significantly in plasma and muscle which prevents $\alpha$-synuclein aggregation in the brain and slows the progression of Parkinson's Disease [29]. Apart from that, it has relieved symptoms like bradykinesia, tremor, rigidity along with instability and balance disorder. Studies show that these neuroprotective therapies do offer symptomatic relief and assist in terms of reversing the progression of the disease. Active exercise incorporates the principle of improving the muscle coordination, stability, balance, hand-eye coordination, reduces muscle rigidity and stiffness [30]. Studies also show that these exercises offer patients suffering from Parkinson's Disease with symptomatic relief in terms of decreased dyskinesia and improved blood circulation. Similarly, another procedural therapy termed FSR - Forceless Spontaneous Release named Yin Tuina is beneficial for PD patients in terms of diminishing clinical symptoms of Parkinson's Disease and improving health \& fitness [31,32].

Meditation also plays a crucial role in terms of releasing dopamine from the striatum. Studies conducted by researchers suggest that meditation helps in restoring the grey matter [33]. It has potential neurological benefits such as increasing grey matter volume along with remarkable improvements in assorted regions of the brain [34]. Apart from that, meditation with regular exercise helps in facilitating long-term benefits such as reducing stress, frustration $\&$ anxiety among PD patients. As per recent studies, it is seen that participants suffering from Parkinson's Disease benefited after 6 months post group meditation program in terms of positive improvement in activities of daily life [35]. Exercise has reduced clinical symptoms of PD like stooped posture, rigidity, aches, monotonous soft voice, and bradykinesia. They make patients less susceptible towards frequent falls, urinary incontinence, postural hypotension and constipation eventually leading towards reduced depression \& fatigue. It improves quality of life \& raises self-esteem. Hence, lifestyle programs like exercises and meditation would be favorable for patients suffering from Parkinson's Disease [8-10].

\section{Effective Recovery with Exercise in Parkinson's Disease}

Regular exercise has shown remarkable progress in patients suffering from Parkinson's Disease (PD). It improves emotional well being as well as motor functions. PD is characterized by bradykinesia, postural abnormalities, resting tremor along with muscular rigidity [36]. It is estimated that more than 10 million people are suffering with PD with approximately 1 million PD patients in U.S. with current limitations in pharmacological and surgical treatments [37]. It is important to identify certain preventive strategies along with supplementary treatment which is more viable in terms of reducing the emotional stress endured by PD patients.

Meditation is one of them, which is highly viable for the healing process as it restores faith and helps the patient to overcome emotional anxiety and nervousness [38]. It helps to restore control movements in PD patients with reduced tremors and balanced energy flow through brain vibration chanting. Apart from that, it is seen that it reduces neurodegeneration in PD patients through precise mechanisms at the molecular level. As per research conducted, it is seen that exercises such as walking on a treadmill, standing and balancing along with exercises which neural electrical impulses can reduce mitochondrial dysfunction and oxidative stress which renders the DJ-1 gene inactive [39]. DJ-1 is a multifunction gene associated as the causative gene for familial protein coding gene PARK7. It maintains mitochondrial \& transcriptional regulations along with antioxidant stress reaction. DJ-1 gene plays a crucial role in terms of patients suffering from sporadic Parkinson's Disease. It is observed that DJ-1 inactivation is linked to increased oxidative stress associated with the pathogenesis of sporadic Parkinson's Disease $[40,41]$.

Several studies suggest that exercise should be incorporated as a comprehensive treatment for PD as it is beneficial and clinical observation shows remarkable progress. Case studies of therapies conducted on PD patients show improved motor skills enhanced self perceived capabilities [42]. Exercise interventions helped PD patients in motor learning along with goal based motor skill training. Patients showed high cognitive skills with improved blood flow and behavioral functions. 


\section{Advances in Pharmacology and Clinical Trials}

Exercises involve improvement in terms of automatic and cognitive components of motor controls with induced effects [43].

\section{Neurorehabilitation and Oxidative Stress Reduction}

Exercise contributes to the general improvement and repair through increased blood flow and promotion of synaptic neuroplasticity. It alleviates motor deficits and focuses mainly on Neurorehabilitation through reduction of oxidative stress and prevents inactivation of DJ-1 novel oncogene [44]. Mutation in DJ-1 or PARK7 can eventually lead to degenerative autosomal hereditary Parkinson's Disease [45]. So, it is important for PD patients to restore some degree of basal ganglia circuitry through exercises directed towards improving neuroplastic mechanisms, behavioral performance and motor learning. Motor deficits of PD patients can be alleviated by incorporating goal based exercise regime which involves medical qigong, standing \& balancing exercises, meditation, Jin Shin Jyutsu \& Yin Tui Na [46-49]. Qigong is a traditional form of physiotherapy with a wide range of complementary methods to treat PD patients [50]. Its application involves a multi dimensional approach with sustained effects on motor and non-motor symptoms of Parkinson's Disease. While, Jin Shin Jyutsu is an ancient art of healing and harmonizing the body through light touch over specific areas of the body. It's quite similar to acupressure, but in an innate and harmonious way. It addresses the issues related to emotional wellbeing, anxiety, nervousness and assists body to restore balance and energy. It alleviates pain and rejuvenates the mind and body through natural healing ability [51]. Lastly, Yin Tui Na massage followed by acupressure is quite effective as it improves tolerability, efficacy and quality of life [52]. Recent studies show that such alternate therapies performed on PD patients with worsening symptoms have shown remarkable progress in terms of motor improvement and reduction of depression in PD patients. It is a form of Chinese energy massage which is performed on the foot corresponding to the affected side of the PD patient. Overall, studies shows that these exercises performed over due course of time has shown prevention of cognitive impairment with improvement in brain function, motor behavior and connectivity. Identification of these novel therapies are quite crucial which can act as a supplementary treatment to Parkinson's Disease $[53,54]$.

Studies show that intensive \& strenuous exercises are related to neurorestorative and neuroprotective features with reduced risk of Parkinson's Disease. It is important to understand the role of exercise in overall brain health which can influence physiological and structural properties of brain function. Neuroplasticity involves a wide range of physiological and structural mechanisms through which brain learns new behaviors and encodes new experiences. Exercises induce repair and strengthening of neuronal circuitry [55].

\section{Neuroprotective Role of DJ-1 Gene}

DJ-1 is a multifunctional protein whose loss of function can trigger progressive neurodegenerative disease like Parkinson's Disease (PD). As per studies conducted, it was found that familial PD can play a significant role in the pathogenesis of sporadic PD [56]. The DJ-1 gene was detected as the causative gene for familial PD PARK7 associated with recessive inheritance. Although, familial PD accounts for ten percent of total PD cases worldwide, but it offers noteworthy insight into the molecular mechanisms of PD onset and symptom manifestation. DJ1 is expressed in the majority of cells and tissues, including Galileo \& neuronal cells. It is important to note that in neurodegenerative diseases like PD, specifically sporadic Parkinson's Disease, DJ-1 is over expressed in reactive astrocytes. Deregulation of Mitochondrial Complex I occurs due to knockdown of DJ-1 expression resulting in oxidative stress and impairment of abstract mediated neuroprotection [57].

DJ-1 is associated with mitochondrial regulation, antioxidative stress reaction, transcriptional regulation and chaperone. It is observed that DJ-1 can be secreted in various cultured cells and tissues and it is located in mitochondria, cytoplasm and nucleus of cells. It should be noted that DJ-1 can undergo nuclear translocation from cytoplasm to nucleus upon exposure to certain stimuli like oxidation and growth factors. Moreover, certain cysteine residues in DJ-1 are susceptible to oxidative stress, such as C106 as it can be oxidized as SO3H eventually leading to loss of DJ-1 functions. As per studies conducted, inactivated DJ-1 with $\mathrm{SO3H}$ and $\mathrm{C} 106$ is observed in PD patients [58,59].

DJ-1 is associated with regulation of DNA-binding transcriptional factors which acts as activators or corepressor. Transcriptional factor activity is regulated by DJ-1 androgen receptor which involves sterol regulatory element binding protein (SREBP), polypyrimidine tract binding protein associated splicing factor (PSF) and nuclear factor erythroid - 2 - related factor 2 (Nrf2) $[60,61]$. Regulation of Nrf2, P53 and PSF is quite important as oxidative stress can have considerable effect 


\section{Advances in Pharmacology and Clinical Trials}

in localizing Nrf2 in cytoplasm and eventual degradation of the ubiquitin - proteasome system. During oxidation stress, DJ-1 can decrease the ROS level through activation of antioxidant stress genes and translocation of Nrf2 into the nucleus of such genes [62].

Most importantly, DJ-1 binds to p53, which results in the regulation of mitochondrial homeostasis against oxidative stress through induction of apoptosis in cells. Apart from that, DJ-1 stimulates the expression of superoxide dismutase (SOD 3) and glutathione ligase gene in order to reduce Reactive Oxygen Species (ROS) level. DJ-1 also up regulates the enzymatic activities of L-Dopa carboxylase (DDC) and Tyrosine Hydroxylase (TH) which synthesizes Dopamine through conversion of L-DOPA to Dopamine $[63,64]$. It critically shows the reason for late onset of Parkinson's Disease in majority of the cases as the activity of DJ-1 towards DDC and TH reduces with aging. Vesicular Monoamine Transporter 2 (VMAT2) is responsible for packing Dopamine in synaptic vesicles while DJ-1 positively regulates VMAT2 gene through protein-protein interaction and transcriptional coactivator. VMAT2 is quite crucial as it prevents neurons from oxidized dopamine induced damage through excess uptake of dopamine in synaptic vesicles. Any heterogeneous or homogenous mutation of DJ-1 can lead to reduced activation of Vesicular Monoamine Transporter 2 (VMAT2), Tyrosine Hydrolase (TH) and LDopa Carboxylase (DDC) which will eventually lead to Parkinson's Disease due to highly oxidized DJ-1 leading inactivation of DJ-1 [65].

\section{Reduction of Neurochemical Deficits and Recovery with Exercise}

Exercise is highly recommended for patients suffering with PD as it reduces aggregation of $\alpha$-synuclein, improves cognitive and motor functions, increases neuroprotective effects, reduces neurochemical deficits and significantly increases DJ-1 protein concentration in muscle \& plasma [66]. Studies suggest that the DJ-1 protein is quite important for normal motor activities. Higher DJ-1 concentration significantly reduces the risk of progression of Parkinson's Disease with less $\alpha$-synuclein aggregation in the brain. Exercise plays a crucial role in terms of altering the progression of the disease with increased DJ-1 concentration [67].

Gene mutations can lead to gain of toxic functions like $\alpha$-synuclein and loss of neuroprotective functions like PINK1 \& DJ-1 [68]. Studies show that DJ-1 gene protects everyone from developing early onset of autosomal recessive Parkinson's Disease. While, mutation related to amino acid in $\alpha$-synuclein gene and gene triplication is related to autosomal-dominant Parkinson's Disease. DJ-1 is also associated with regulation of H1F1 transcriptional activity under hypoxic conditions along with up regulation of Hsp70 in order to prevent abnormal protein aggregation [69]. Overall, voluntary exercises can prevent the decline of age related cognitive and motor abilities by up regulating DJ-1 which will eventually stop or slow the progression of Parkinson's Disease. It is noteworthy to note that due to limitation of drug treatment, PD patients should enroll in an intensive exercise program to increase DJ-1 levels, prevent $\alpha$-synuclein aggregation and eventually lead to the normal motor functioning ability of PD patients [70].

\section{Conclusion}

Parkinson's Disease is a progressively degenerative disease. It is studied that active exercise significantly reduces the loss of neurons with lesser aggregation of alpha-synuclein and Lewy body in the brain. Moreover, it improves motor and cognitive function with increased DJ1 protein concentration. Upregulation of $\mathrm{DJ}-1$ protein which can cause a reduction in severity of PD symptoms and lower $\alpha$-synuclein accumulation in notable parts of the brain. It is recommended that in order to address the chronic and progressive degenerative disorder like PD, patients should exercise regularly with meditation and brain vibration chanting therapy. It offers neuroprotection and significantly improves motor function which may slow the progression of Parkinson's Disease.

\section{References}

1. Lakso Merja, Vartiainen S, Moilanen AM, Sirviö J, Thomas JH, et al. (2003) Dopaminergic neuronal loss and motor deficits in Caenorhabditis elegans overexpressing human $\alpha$-synuclein. J Neurochem 86(1): 165-172.

2. Hirsch Etienne, Ann M Graybiel, Yves A Agid (1988) Melanized dopaminergic neurons are differentially susceptible to degeneration in Parkinson's disease. Nature 334(6180): 345-348.

3. Wakabayashi Koichi, Hayashi S, Kakita A, Yamada M, Toyoshima Y, et al. (1998) Accumulation of $\alpha$ synuclein/NACP is a cytopathological feature common to Lewy body disease and multiple system atrophy. Acta Neuropathol 96(5): 445-452. 


\section{Advances in Pharmacology and Clinical Trials}

4. Kempster PA, Frankel JP, Bovingdon M, Webster R, Lees AJ, et al. (1989) Levodopa peripheral pharmacokinetics and duration of motor response in Parkinson's disease. J Neurol Neurosurg Psychiatry 52(69): 718-723.

5. Han L, Xie YH, Wu R, Chen C, Zhang Y, et al. (2017) Traditional Chinese medicine for modern treatment of Parkinson's disease. Chin J Integr Med 23(8): 635640 .

6. Pathak-gandhi N, Vaidya AD (2016) Management of Parkinson's disease in Ayurveda: Medicinal plants and adjuvant measures. J Ethnopharmacol 197: 46-51

7. Walton-hadlock J (1999) Primary Parkinson's disease: the use of Tuina and acupuncture in accord with an evolving hypothesis of its cause from the perspective of Chinese traditional medicine--Part 2. Am J Acupunct 27(1-2): 31-49.

8. Smart K, Durso R, Morgan J, McNamara P (2016) A potential case of remission of Parkinson's Disease. J Complement Integr Med 13(3): 311-315.

9. Last N, Tufts E, Auger LE (2017) The Effects of Meditation on Grey Matter Atrophy and Neurodegeneration: A Systematic Review. J Alzheimers Dis 56(1): 275-286.

10. Advocat J, Enticott J, Vandenberg B, Hassed C, Hester J, et al. (2016) The effects of a mindfulness-based lifestyle program for adults with Parkinson's disease: a mixed methods, wait list controlled randomised control study. BMC Neurol 16:166.

11. Prakash J, Chouhan S, Yadav SK, Westfall S, Rai SN, et al. (2014) Withaniasomnifera alleviates parkinsonian phenotypes by inhibiting apoptotic pathways in dopaminergic neurons. Neurochem Res 39(12): 2527-2536.

12. Yang Y, Qiu WQ, Hao YL, Lv ZY, Jiao SJ, et al. (2015) The efficacy of traditional Chinese Medical Exercise for Parkinson's disease: a systematic review and meta-analysis. PLoS One 10(4): e0122469.

13. McCormack AL, Thiruchelvam M, Manning-Bog AB, Thiffault C, Langston JW, et al. (2002) Environmental risk factors and Parkinson's disease: selective degeneration of nigral dopaminergic neurons caused by the herbicide paraquat. Neurobiol Dis 10(2): 119127.
14. Bloem BR, Van Vugt JP, Dennis J Beckley (2001) Postural instability and falls in Parkinson's disease. Adv Neuro l 87: 209-223.

15. Sunil A, Raghavendra Rao MV, Gaurang Prabhu, Sanju Aggarwal, David A, et al. (2016) Blinded Independent Central Imaging in Clinical Trials: Does it ensure Against Bias? Canc Therapy \& Oncol Int J 1(1): 1-2.

16. Missale Cristina, Nash SR, Robinson SW, Jaber M, Caron MG (1998) Dopamine receptors: from structure to function. Physiol Rev 78(1): 189-225.

17. Wolff JA, Fisher LJ, Xu L, Jinnah HA, Langlais PJ, et al. (1989). Grafting fibroblasts genetically modified to produce L-dopa in a rat model of Parkinson disease. Proceedings of the National Academy of Sciences 86(22): 9011-9014.

18. Geraghty JJ, Jankovic J, Zetusky WJ (1985) Association between essential tremor and Parkinson's disease. Annals of Neurology 17(4): 329-333.

19. Yahr MD, Duvoisin RC, Schear MJ, Barrett RE, Hoehn MM (1969) Treatment of parkinsonism with levodopa. Archives of neurology 21(4): 343-354.

20. Nardone Antonio, Marco Schieppati (2006) Balance in Parkinson's disease under static and dynamic conditions. Movement disorders 21(9): 1515-1520.

21. Yahr Melvin D, Duvoisin RC, Schear MJ, Barrett RE, Hoehn MM (1969) Treatment of parkinsonism with levodopa. Archives of Neurology 21(4): 343-354.

22. Singleton $A B$, Farrer $M$, Johnson J, Singleton A, Hague $S$, et al. (2003) $\alpha$-Synuclein locus triplication causes Parkinson's disease. Science 302(5646): 841.

23. Masliah E, Rockenstein E, Veinbergs I, Sagara Y, Mallory M, et al. (2001) $\beta$-Amyloid peptides enhance $\alpha$-synuclein accumulation and neuronal deficits in a transgenic mouse model linking Alzheimer's disease and Parkinson's disease. Proc Natl Acad Sci U S A 98(21): 12245-12250.

24. Sunil A, Sanju A, Somya AA (2016) Study of Lean in Oncology: Reducing Waste and Increasing Value. Canc Therapy \& Oncol Int J 1(3): 1-4.

25. Tofaris George K., Garcia Reitböck P, Humby T, Lambourne SL, O'Connell M, et al. (2006) Pathological changes in dopaminergic nerve cells of the substantia nigra and olfactory bulb in mice transgenic for 


\section{Advances in Pharmacology and Clinical Trials}

truncated human $\alpha$-synuclein (1-120): implications for Lewy body disorders. J Neurosci 26(15): 39423950.

26. Gibb WR, Lees AJ (19998) The relevance of the Lewy body to the pathogenesis of idiopathic Parkinson's disease. J Neurol Neurosurg Psychiatry 51(6): 745752 .

27. Betarbet Ranjita, , Sherer TB, MacKenzie G, GarciaOsuna M, Panov AV, et al. (2000) Chronic systemic pesticide exposure reproduces features of Parkinson's disease. Nat Neurosci 3(12): 1301-1306.

28. Cristian Adrian, Katz M, Cutrone E, Walker RH (2005) Evaluation of acupuncture in the treatment of Parkinson's disease: a double-blind pilot study. Mov Disord 20(9): 1185-1188.

29. Zhou W, Barkow JC, Freed CR (2017) Running wheel exercise reduces $\alpha$-synuclein aggregation and improves motor and cognitive function in a transgenic mouse model of Parkinson's disease. PLoS ONE 12(12): e0190160.

30. Rocchi L, Chiari L, Horak FB (2002) Effects of deep brain stimulation and levodopa on postural sway in Parkinson's disease. J Neurol Neurosurg Psychiatry 73(3): 267-274.

31. Schmitz-Hübsch Tanya, Pyfer D, Kielwein K, Fimmers R, Klockgether T, et al. (2006) Qigong exercise for the symptoms of Parkinson's disease: a randomized, controlled pilot study. Mov Disord 21(4): 543-548.

32. Eng ML, Lyons KE, Greene MS, Pahwa R (2006) Openlabel trial regarding the use of acupuncture and yin tuina in Parkinson's disease outpatients: a pilot study on efficacy, tolerability, and quality of life. J Altern Complement Med 12(4): 395-399.

33. Pagnoni Giuseppe, Milos Cekic (2007) Age effects on gray matter volume and attentional performance in Zen meditation. Neurobiol Aging 28(10): 1623-1627.

34. Newberg AB, Serruya M, Wintering N, Moss AS, Reibel D, et al. (2014) Meditation and neurodegenerative diseases. Annals of the New York Academy of Sciences 1307(1): 112-123.

35. Pickut BA, Van hecke W, Kerckhofs E, Mariën $P$, Vanneste S, et al. (2013) Mindfulness based intervention in Parkinson's disease leads to structural brain changes on MRI: a randomized controlled longitudinal trial. Clin Neurol Neurosurg 115(12): 2419-25.

36. Radak Z, Chung HY, Goto S (2008) Systemic adaptation to oxidative challenge induced by regular exercise. Free Radic Biol Med 44(2): 153-159.

37. Strickland D, Bertoni JM (2004) Parkinson's prevalence estimated by a state registry. Movement disorders: official journal of the Movement Disorder Society 19(3): 318-323.

38. Fitzpatrick L, Simpson J, Smith A (2010) A qualitative analysis of mindfulness-based cognitive therapy (MBCT) in Parkinson's disease. Psychol Psychother 83(2): 179-192.

39. Canet-Avilés RM, Wilson MA, Miller DW, Ahmad R, McLendon C, et al. (2004) The Parkinson's disease protein DJ-1 is neuroprotective due to cysteinesulfinic acid-driven mitochondrial localization. Proceedings of the National Academy of Sciences, 101(24): 9103-9108.

40. Abou-Sleiman PM, Healy DG, Quinn N, Lees AJ, Wood NW (2003) The role of pathogenic DJ-1 mutations in Parkinson's disease. Ann Neurol 54(3): 283-286.

41. Bonifati V, Rizzu P, van Baren MJ, Schaap 0, Breedveld GJ, et al. (2003) Mutations in the DJ-1 gene associated with autosomal recessive early-onset parkinsonism. Science 299(5604): 256-259.

42. Hughes AJ, Daniel SE, Kilfor, L, Lees AJ (1992) Accuracy of clinical diagnosis of idiopathic Parkinson's disease: a clinico-pathological study of 100 cases. J Neurol Neurosurg Psychiatry 55(3): 181184.

43. Goodwin VA, Richards SH, Taylor RS, Taylor AH, Campbell JL (2008) The effectiveness of exercise interventions for people with Parkinson's disease: A systematic review and meta-analysis. Mov disord 23(5): 631-640.

44. Kahle PJ, Waak J, Gasser T (2009) DJ-1 and prevention of oxidative stress in Parkinson's disease and other age-related disorders. Free Radic Biol Med 47(10): 1354-1361.

45. Bandopadhyay R, Kingsbury AE, Cookson MR, Reid AR, Evans IM, et al. (2004) The expression of DJ-1 (PARK7) in normal human CNS and idiopathic Parkinson's Disease. Brain 127(2): 420-430. 


\section{Advances in Pharmacology and Clinical Trials}

46. Da Silva GD, Lorenzi-Filho G, Lage LV (2007) Effects of yoga and the addition of Tui $\mathrm{Na}$ in patients with fibromyalgia. The J Altern Complement Med 13(10): 1107-1114.

47. Crohn Steven. Apparatus and method for the point treatment of a patient by acupuncture and light. U.S. Patent Application No. 11/169,371.

48. Salach MD (2006) The effects of Reiki, a complementary alternative medicine, on depression and anxiety in the Alzheimer's and dementia population. Unpublished Master of Arts in Gerontology, San Francisco State University, San Francisco.

49. Walton-Hadlock J (1999) Primary Parkinson's disease: the use of Tuina and acupuncture in accord with an evolving hypothesis of its cause from the perspective of Chinese traditional medicine--Part 2. Am J Acupunct 27(1-2): 31-49.

50. Schmitz-Hübsch T, Pyfer D, Kielwein K, Fimmers R, Klockgether T, et al. (2006) Qigong exercise for the symptoms of Parkinson's disease: a randomized, controlled pilot study. Mov disord 21(4): 543-548.

51. Moyer CA, Rounds J, Hannum JW (2004) A metaanalysis of massage therapy research. Psychol Bull 130(1): 3.

52. Huang Y, Jiang X, Zhuo Y, Tang A, Wik G (2009) Complementary acupuncture treatment increases cerebral metabolism in patients with Parkinson's disease. Int J Neurosci 119(8): 1190-1197.

53. Goetz CG, Fahn S, Martinez-Martin P, Poewe W, Sampaio C, et al. (2008) Movement Disorder Society-sponsored revision of the Unified Parkinson's disease Rating Scale (MDS-UPDRS): scale presentation and clinimetric testing results. Movement disord 23(15): 2129-2170.

54. Hirsch MA, Farley BG (2009) Exercise and neuroplasticity in persons living with Parkinson's disease. Eur J phys Rehabil Med 45(2): 215-229.

55. Moore DJ, West AB, Dawson VL, Dawson TM (2005) Molecular pathophysiology of Parkinson's disease. Annu Rev Neurosci 28: 57-87.

56. Zhang L, Shimoji M, Thomas B, Moore DJ, Yu SW, et al. (2005) Mitochondrial localization of the Parkinson's disease related protein DJ-1: implications for pathogenesis. Hum Mol Genet 14(14): 2063-2073.

57. Ariga H, Takahashi Niki K, Kato I, Maita H, Niki T, et al. (2013) Neuroprotective function of DJ-1 in Parkinson's Disease. Oxid med cell longev 2013: 683920.

58. Yamaguchi S, Yamane T, Takahashi Niki K, Kato I, Niki $\mathrm{T}$, et al. (2012) Transcriptional activation of lowdensity lipoprotein receptor gene by DJ-1 and effect of DJ-1 on cholesterol homeostasis. PLoS One 7(5): e38144.

59. Gan L, Johnson DA, Johnson JA (2010) Keap1-Nrf2 activation in the presence and absence of DJ-1. Eur J Neurosci 31(6): 967-977.

60. de Vries HE, Witte M, Hondius D, Rozemuller AJ, Drukarch B, et al. (2008) Nrf2-induced antioxidant protection: a promising target to counteract ROSmediated damage in neurodegenerative disease? Free Radic Bio Med 45(10): 1375-1383.

61. Zhong N, Kim CY, Rizzu P, Geula C, Porter DR, et al. (2006) DJ-1 transcriptionally up-regulates the human tyrosine hydroxylase by inhibiting the sumoylation of pyrimidine tract-binding protein-associated splicing factor. J Biol Chem 281(30): 20940-20948.

62. Ishikawa S, Taira T, Niki T, Takahashi Niki K, Maita C, et al. (2009) Oxidative status of DJ-1-dependent activation of dopamine synthesis through interaction of tyrosine hydroxylase and L-DOPA decarboxylase with DJ-1. J Biol Chem 284(42): 28832-28844

63. Chen L, Cagniard B, Mathews T, Jones S, Koh HC, et al. (2005) Age-dependent motor deficits and dopaminergic dysfunction in DJ-1 null mice. J Biol Chem 208(22): 21418-21426.

64. Hong Z, Shi M, Chung KA, Quinn JF, Peskind ER, et al. (2010) DJ-1 and $\alpha$-synuclein in human cerebrospinal fluid as biomarkers of Parkinson's Disease. Brain 133(3): 713-726.

65. Hamamichi S, Rivas RN, Knight AL, Cao S, Caldwell KA, et al. (2008) Hypothesis-based RNAi screening identifies neuroprotective genes in a Parkinson's disease model. Proc Natl Acad Sci 105(2): 728-733.

66. Dodson MW, Guo M (2007) Pink1, Parkin, DJ-1 and mitochondrial dysfunction in Parkinson's disease. Curr opin neurobiol 17(3): 331-337. 


\section{Advances in Pharmacology and Clinical Trials}

67. Batelli S, Albani D, Rametta R, Polito L, Prato F, et al. (2008) DJ-1 modulates $\alpha$-synuclein aggregation state in a cellular model of oxidative stress: relevance for Parkinson's disease and involvement of HSP70. PloS one 3(4): e1884.

68. Gandhi S, Wood NW (2005) Molecular pathogenesis of Parkinson's disease. Hum Mol Genet 14(18): 27492755.

69. Lang AE, Lozano AM (1998) Parkinson's disease. New England Journal of Medicine 339(16): 1044-1053.
70. Betarbet R, Canet Aviles RM, Sherer TB, Mastroberardino PG, McLendon C, et al. (2006) Intersecting pathways to neurodegeneration in Parkinson's disease: effects of the pesticide rotenone on DJ-1, $\alpha$-synuclein, and the ubiquitin-proteasome system. Neurobiol Dis 22(2): 404-420. 